Highly Energetic Physical Processes and

Mechanisms for Emission from Astrophysical Plasmas

IAU Symposium, Vol. 195, 2000

P. C. H. Martens, S. Tsuruta, and M. A. Weber, eds.

\title{
Radio Evidence for Nonthermal Particle Acceleration in Normal Stars
}

\author{
K. R. Lang \\ Department of Physics and Astronomy, Tufts University, Medford, MA \\ 02155, U.S.A.
}

\begin{abstract}
The Very Large Array has been used to detect nonthermal radio emission from nearby stars of late spectral type $F, G, K$, and $M$, and has provided unique high-resolution investigations of the Sun's radio emission.
\end{abstract}

\section{Very Large Array}

The Very Large Array (VLA) has been used to study the Sun and nearby stars at wavelengths from $0.7 \mathrm{~cm}$ to $400 \mathrm{~cm}$. Although this radiation has relatively low photon energy, it is emitted by high-energy electrons, from $10 \mathrm{keV}$ to $1 \mathrm{MeV}$ in energy, and can be used to probe the temperature, electron density, and magnetic field strength and structure in the regions of both nonthermal and thermal particle acceleration.

The large collecting area of the VLA permits the detection of the relatively faint radio emission from nearby main-sequence stars of late spectral type $F, G$, $\mathrm{K}$, and $\mathrm{M}$. This radiation must be nonthermal. The observed radio flux densities can only be provided by thermal emission at the $20-\mathrm{cm}$ wavelength if the stars contain million-degree coronae that are hundreds of times larger that the starwhich is highly implausible. In contrast, the radio Sun is dominated by thermal radiation that would not be detected by the VLA if the Sun was placed at the distance of the next nearest star.

The advantage of the VLA for solar studies is its angular resolution, as low as 0.05 seconds of arc at the $0.7 \mathrm{~cm}$ wavelength, corresponding to a linear scale of 36 kilometers at the solar photosphere. VLA snapshot maps can be obtained with this angular resolution at time intervals of less than one second.

\section{Long-lived ( $>1$ Hour) Stellar Radio Radiation}

The nonthermal, quiescent radio radiation of nearby normal stars of late spectral type $F, G, K$, and $M$ increases in strength with the thermal X-ray luminosity, suggesting that the nonthermal particle acceleration process (radio) is related to the thermal heating (X-ray) of stellar coronae (Güdel \& Benz 1993; Lang 1996).

The long-lived ( $>1$ hour), quiescent radio emission from some of these stars is 100 percent circularly polarized. This polarization is attributed to intense magnetic fields of several hundred gauss in strength in the emitting region. Thus, magnetic fields are most likely involved in the long-lived, nonthermal particle 
acceleration processes on these stars.

The thermal X-ray luminosity of main-sequence stars with late spectral type increases with their rotation speed (Hempelmann et al. 1995), suggesting that enhanced dynamo action by rapid rotation results in stronger magnetic fields and more intense X-ray radiation (also see Pallavicini et al. 1981).

The long-lived radio emission detected from the Sun differs according to wavelength (Lang 1999). At the long $90-\mathrm{cm}$ wavelength, one detects nonthermal noise storms lasting for hours and days, emitted by energetic electrons trapped in large-scale magnetic loops that are several times larger than active regions in size. At short centimeter wavelengths, the VLA has detected nonthermal radiation spectra from X-ray bright points, EUV blinkers, and flares at the edges of the magnetic network (Lang 1999). Coronal heating on the Sun may be attributed to frequent low-level flares at the network boundaries (Krucker \& Benz 1998).

\section{Short-lived ( $<1$ Hour) Stellar Flares}

Dwarf $\mathrm{M}$ flare stars emit powerful, short-lived flares that are 100 percent circularly polarized, implying magnetic fields of several hundred gauss in strength. The rapid rise times of less than 200 milliseconds indicate flaring sources smaller than one-hundredth of the stellar radius, using the argument that nothing can move faster than the velocity of light. In other words, the flaring emission from normal stars originates in a highly structured magnetic corona (Lang 1994). Coherent emission is also required since the brightness temperatures exceed $10^{15} \mathrm{~K}$.

The VLA was used more than a decade ago to show that energetic electrons are accelerated at the apex of coronal loops in active regions (see Lang 1995, 1998). More recently, the VLA has been used to show that the radio emission from Coronal Mass Ejections illuminates large-scale magnetic loops comparable to the Sun in size (Willson et al. 1998).

Acknowledgments. Radio astronomical studies of the Sun at Tufts Univ. are supported by NASA Grants NAG5-4972, NAG5-6269, and NAG5-8155.

\section{References}

Güdel, M., \& Benz, A. O. 1993, ApJ, 405, L63

Hempelmann, A., Schmitt, J. H. M. M., Schultz, M., Ruediger, G., \& Stepien, K. 1995, A\&A, 294, 515

Krucker, S., \& Benz, A. O. 1998, ApJ, 501, L213

Lang, K. R. 1994, ApJS, 90, 753

Lang, K. R. 1995, Sun, Earth and Sky (New York: Springer-Verlag)

Lang, K. R. 1996, in Magnetohydrodynamic Phenomena in the Solar System, eds. Y. Uchida et al. (Boston: Kluwer)

Lang, K. R. 1999, Surveys in Geophysics, 19, 521

Pallavicini, R., Golub, L., Rosner, R., Vaiana, G. S., Ayres, T., \& Linsky, J. L. 1981, ApJ, 248, 279

Willson, R. F., Redfield, S. L., Lang, K. R., Thompson, B. J., \& St. Cyr, O. C. 1998, ApJ, 504, L117 